\title{
Characterization of a highly-segmented silicon prototype for the TRACE array
}

\author{
M. Gelain ${ }^{1, a}$, D. Mengoni ${ }^{2}$, S. Lunardi², J. Dueñas ${ }^{3}$, M. Assie ${ }^{4}$, and the GHT collaboration \\ ${ }^{1}$ Dipartimento di Fisica e Astronomia, Università di Padova, Padova, Italy and INFN, Laboratori Nazionali di \\ Legnaro, Legnaro (Padova), Italy. \\ ${ }^{2}$ Dipartimento di Fisica e Astronomia, Università di Padova, Padova, Italy and INFN, Sezione di Padova, \\ Padova, Italy. \\ ${ }^{3}$ Departamento de Fisica Aplicada, FCCEE Universidad de Huelva, 21071 Huelva, Spain \\ ${ }^{4}$ Institut de Physique Nucléaire, Université Paris-Sud-11-CNRS/IN2P3, 91406 Orsay, France
}

\begin{abstract}
In view of the construction of novel and high-sensitive instrumentation for the emerging ISOL facilities new prototypes have being implemented and tested. The contribution focuses at the investigation of the detection efficiency of an innovative silicon-pad prototype, which is the key element for the construction of the TRACE array, pursued for the SPES facility based at the Legnaro National Laboratories (Italy). The inter-pad size has been estimated by using a commercial 100-MHz-14-bit CAEN digitizer for sampling the signals obtained by an alpha-source scan over the inter-pad region.
\end{abstract}

\section{Introduction}

Nowadays silicon-based detectors have reached an unprecedented level of sophistication which makes such choice an extremely convenient solution for particle-detection application, especially in view of the cutting-edge instrumentation, namely TRACE [1], GASPARD [2] and HYDE [3], required at the new intense ISOL facilities, such as SPES [4] and SPIRAL2 [5].

The silicon approach benefits of the technological boost due to the revolutionary progress in the microelectronics industry. This has resulted in a large variety of shapes and designs, in the 6-inches wafer technology and with sub- millimetre electrode segmentation, and an outstanding uniformity of the substrate, due to the neutron- transmutation process. Nonetheless some limitations still affect the design of any common silicon-based detector, among which primarily: the radiation damage and the efficiency loss due to the inter-strip and dead-layer presence. To characterize the second aspect in the new highly-segmented silicon detector for charged particles and light ions, a source test was performed at LNL. The obtained results have been afterwards compared with a simulation.

\section{Experimental set-up}

A highly segmented TRACE detector, dimensions of $2.5 \times 2 \mathrm{~cm}^{2}$ and in 30 pads, $4 \mathrm{~mm} \mathrm{x} 4 \mathrm{~mm}$ each, was chosen for the alpha-source test experiment. The detector was operated in a vacuum system, consisting

\footnotetext{
a e-mail: Michele.Gelain@lnl.infn.it
} 
of chamber, a fore-vacuum pump (range: $\sim 10^{-2}$ mbar) vacuum gauges and a Pirani-Penning Gauge (range: 1000 mbar $\div 5 \cdot 10^{-9}$ mbar). A single-peak alpha-calibration source of ${ }^{241} \mathrm{Am}$ was collimated by using a steel cone, having a hole of $(0.10 \pm 0.05) \mathrm{mm}$ diameter. The set-up can be seen in Fig. 1.

\section{Measurements and interpretation}

The experiment aims two final goals:

- The estimation of the active area of the detector, by measuring the inter-pad gap.

- The estimation of the efficiency loss by investigating the neighbouring-pad correlation.

For the first scope an inter-pad scan was performed with a step of $30 \mu \mathrm{m}$, taking as an observable the integral of the energy peak. Therefore the inter-pad area was estimated by considering the distance where the intensity was decreasing from $90 \%$ to $10 \%$, green line or, vice versa, red line in Fig. 2. Between two consecutive pads, neglecting the edges of the detector, the inter-pad size is $(60 \pm$ 6) $\mu \mathrm{m}$ : the active zone is thus reduced by $1.5 \%$.

In addition a charge loss effect is also visible, as the experimental points do not sum to one, underneath the inter-pad region. This is presumably due to the charge trapping effect and the presence of a weak electric field.

The waveforms, $50-\mu \mathrm{s}$ long, collected by $1-\mathrm{MHz}$ 14-bits digitizers, have been filtered, by using a trapezoidal filter, editable by the experimenter.
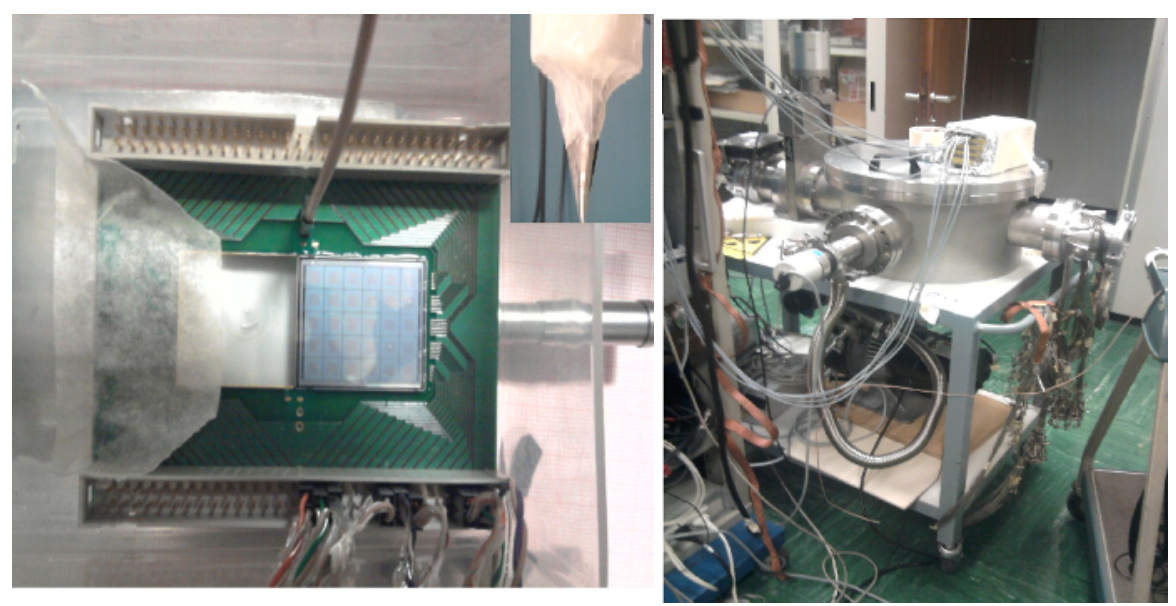

Figure 1. On the Left, a prototype of the TRACE pad detector on a micrometer scanning system is visible. The steel cone used as a collimator is also presented in the inset. On the right, the experimental setup, with the chamber and the preamplifier in foreground.

For the interpretation we compare the data with a simulation [6], which takes into account the distortion of the electric field and the motion of the charges in the semiconductor. After accumulating sufficient statistics, the signals were sorted in a correlation matrix, visible in Fig. 3. 


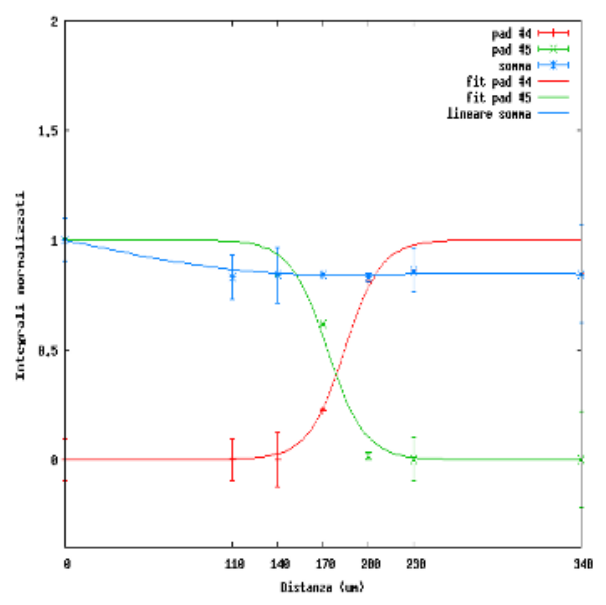

Figure 2. Normalized intensity as a function of the position during the scan. The zero was chosen arbitrarily to have the collimating source in the center of a pad. The lines are obtained by a Fermi-Dirac function.

The simulation presented in Fig. [3] shows that we can distinguish four distinct areas and four vertices where the holes and electrons behave differently.

With reference to the Fig. 3, in A and $\mathrm{G}$ all the holes move to the nearest strip and all electrons move to $\mathrm{N}$-side; in $\mathrm{B}$ and $\mathrm{F}$, all the holes move to the nearest strip and electrons are shared between $\mathrm{SiO}_{2}$ and $\mathrm{N}$-side. All the holes move to the nearest strip and all electrons move to $\mathrm{SiO}_{2}$ in $\mathrm{C}$ and E. Holes are shared between strips and all electrons move to $\mathrm{SiO}_{2}$ in $\mathrm{D}$, due to an accumulation of positive charge in the oxide. Finally charge-sharing is taking place between $\mathrm{G}$ and $\mathrm{A}$.

In our source test, the events in zone $\mathrm{H}$ are almost as the incident particles do not penetrate deep enough to produce any significant charge-sharing effect. Furthermore, the application of the trapezoidal filters is optimized for the signals of a defined rise time and this leads to an incorrect energy derivation whenever signals are faster or slower. As a consequence in the graph, the zones B and F are more inclined expected from simulation.

A deeper understanding of the origin of the various regions requires the investigation of the signals in coincidence with such events. This enables a more convincing comparison with the conclusions drawn from the simulation. To this end a selection of the various loci has been applied and the corresponding signal, polarity and amplitude, match with the simulated results.

A notable diffrence between the experimental and the simulated data is the presence of points with decreasing energy along the positive $\mathrm{X}$ and $\mathrm{Y}$ axis. This is likely due to a partial charge collection in the inter-pad region.

\section{Conclusions and perspectives}

In conclusion, the experiment allowed to estimate a reduction of about $1.5 \%$ in the active area of the detector, when considering a pad distant from the external guard ring. In addition it can be concluded that the efficiency is recoverable only under certain conditions, i.e. whenever the energies are known. The overall result is consistent with a simulation outcome, confirmed as well by the investigation of the digitized waveforms. 

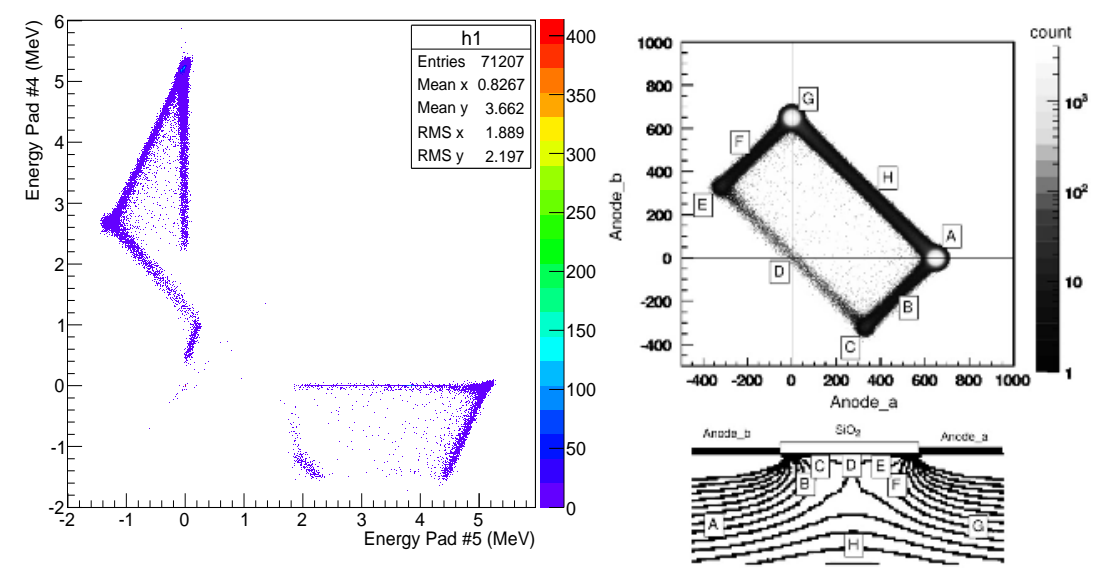

Figure 3. Energy correlation matrix among two neighboring pads. The matrix shows two important zones: the zone of the maximum energy for each pad, recognizable from the scale intensity and the charge-sharing zone, the lines almost parallel to the first bisector. On the top right, the simulation [6] of the correlation matrix between the energy of two neighboring pads. On the bottom right, the calculation of the electric field, where the four regions and vertices of the correlation matrix are spot. The presence of points along the $\mathrm{X}$ and $\mathrm{Y}$ axis, at an energy lower than the alpha peak, is likely to be ascribed to a charge loss in the inter-pad region.

In future we plan to improve the trapezoidal filter and compare the results with a simplified simulation approach. In addition we also plan to make a measurement of the pad resistivity.

\section{Acknowledgments}

The author is grateful to the GHT collaboration. This work has been supported by the Istituto Nazionale di Fisica Nucleare (Italy), and by the Ministero dell'Istruzione, Università e Ricerca (MIUR) under the program FIRB08, project C91J10000110001.

\section{References}

[1] A. Gadea et al., Nucl. Ins. Meth. A654, 88-96 (2011).

[2] http://gaspard.in2p3.fr/index.html

[3] I. Martel et al., Proceedings of the DAE Symp.on Nucl. Phys. 55 (2010)

[4] http://web.infn.it/spes

[5] http://www.ganil-spiral2.eu/spiral2

[6] S. Takeda et al., Nucl. Ins. Meth. A579, 859-865 (2007). 